\title{
Flow structures govern particle collisions in turbulence
}

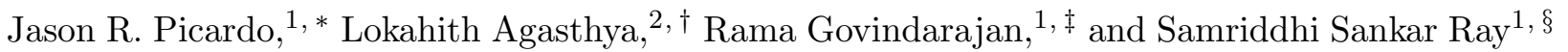 \\ ${ }^{1}$ International Centre for Theoretical Sciences, Tata \\ Institute of Fundamental Research, Bangalore 560089, India \\ ${ }^{2}$ Indian Institute for Science Education and Research, Pune 411008, India
}

\begin{abstract}
The role of the spatial structure of a turbulent flow in enhancing particle collision rates in suspensions is an open question. We show and quantify, as a function of particle inertia, the correlation between the multiscale structures of turbulence and particle collisions: Straining zones contribute predominantly to rapid head-on collisions compared to vortical regions. We also discover the importance of vortex-strain worm-rolls, which goes beyond ideas of preferential concentration and may explain the rapid growth of aggregates in natural processes, such as the initiation of rain in warm clouds.
\end{abstract}

Turbulence is riddled with a hierarchy of interacting vortical and straining structures (Fig. 1), which are closely related to its characteristic intermittent and non-Gaussian statistics [1 6]. The most intense structures typically occur near each other, in the form of vortex tubes surrounded by straining sheets, as shown in Fig. 1. This organization - a sort of vortex-strain worm-rolls - is characteristic of turbulent flows [7-10], and its origin and dynamical implications continue to be investigated [11 16]. These structures distinguish fully developed turbulence from purely random flow fields, and must play an important role in many aspects of turbulent transport. The most important of these - because it remains central to our understanding of phenomena as diverse as the formation of planets in circumstellar disks [17] or the initiation of rain in warm clouds [18, 19] is the growth of macroscopic aggregates, due to collisions and coalescences, from nuclei-particles (dust or aerosols) suspended in a turbulent flow. The role of the underlying turbulent carrier flow is critical: Estimates of, e.g., the rate of growth of these aggregates in the absence of such flows do not agree with that seen in nature [20]. Indeed, the explanation of such rapid growth through coalescence, demonstrated [21,23] and quantified in terms of flow statistics [20, 24, 25], is rooted in the ability of turbulent flows to enhance the rate of collisions between nuclei seed-particles.

A critical discovery, due to Bec, et al. [22, was to find the precise connection between the intermittent (multiscaling) nature of the carrier turbulent flow and the accelerated growth of aggregates. And yet the implied correlation between the structure of the flow and droplet collisions-coalescences remains unknown. Indeed, there is evidence to suggest that flow structures matter. But the question is how and when?

In this paper we answer this question comprehensively, based on direct numerical simulations (DNSs), and show how straining regions are intrinsically more effective at generating collisions than vortical ones even for uniformly distributed inertia-less particles. Particle inertia widens this discrepancy, not simply by preferential concentration, but also by selectively increasing the collision velocities in straining zones. This is because straining regions have a larger proportion of head-on or rear-end collisions, as opposed to side-on collisions, which are predominant in vortical regions. Consequently, a larger fraction of the velocity gradient in straining zones is translated into the particle approach velocity. Finally, and most strikingly, we show how intense vorticity and strain, cohabiting as vortex-strain worm-rolls, conspire to generate rapid, violent collisions.

We therefore consider an incompressible $(\nabla \cdot \boldsymbol{u}=0)$ turbulent flow whose velocity $\boldsymbol{u}$ is a solution to the Navier-Stokes equation

$$
\partial_{t} \boldsymbol{u}+(\boldsymbol{u} \cdot \nabla) \boldsymbol{u}=-\nabla p+\nu \nabla^{2} \boldsymbol{u}+\boldsymbol{f}
$$

* jrpicardo@icts.res.in; picardo21@gmail.com

† lokahith.agasthya@students.iiserpune.ac.in

¥ rama@icts.res.in

$\S$ samriddhisankarray@gmail.com 


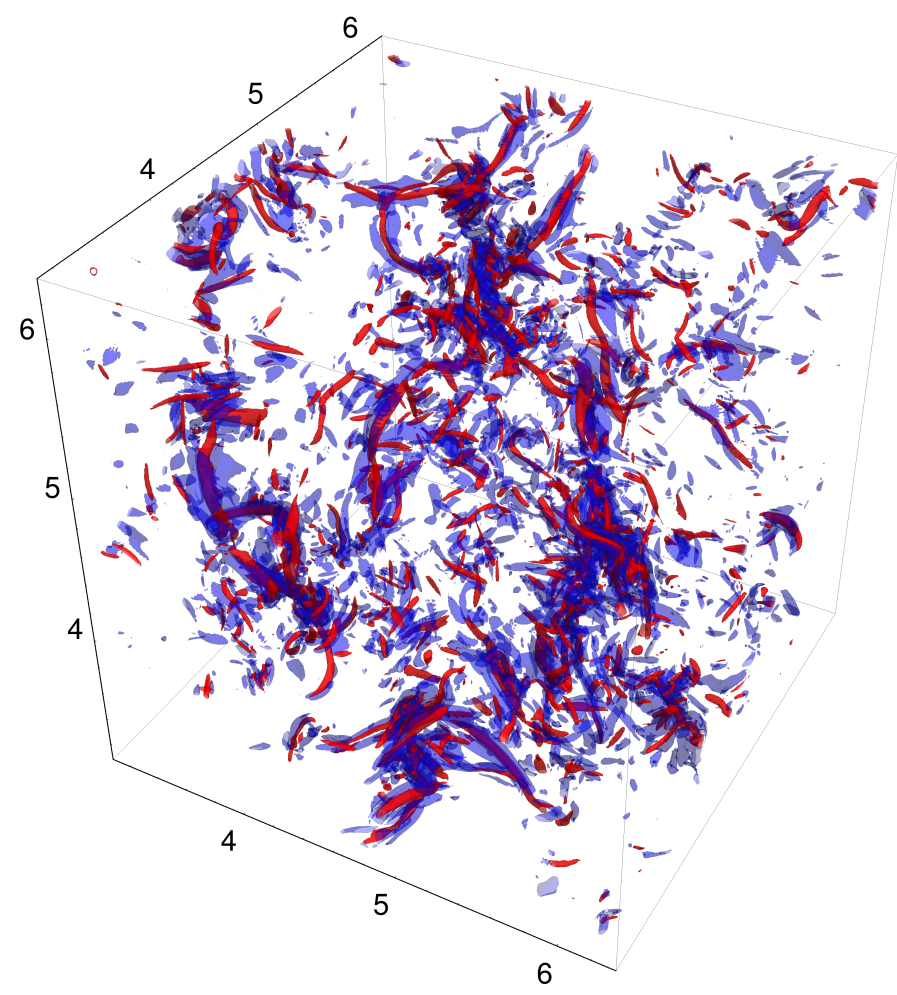

FIG. 1. A representative snapshot of three-dimensional contours of $\mathcal{Q}$ showing intense vortex tubes (opaque red: $+5.6 \sqrt{\left\langle\mathcal{Q}^{2}\right\rangle}$ ) enveloped by dissipative, straining sheets (transparent blue: $-2 \sqrt{\left\langle\mathcal{Q}^{2}\right\rangle}$ ).

where $\nu$ is the kinematic viscosity. We perform DNSs on a tri-periodic domain with $N^{3}=512^{3}$ grid points, by using a standard de-aliased pseudo-spectral solver [26] and a second-order AdamsBashford scheme for time-integration. A statistically stationary, homogeneous and isotropic flow is maintained by the time-dependent large-scale forcing $\boldsymbol{f}$, which injects a constant amount of energy, and hence dissipation $\epsilon$, into the first two wavenumber shells. The Kolmogorov length $\eta=\left(\nu^{3} / \epsilon\right)^{1 / 4}$ satisfies $\eta k_{\max } \approx 1.7$ (where $k_{\max }=\sqrt{2} N / 3$ is the maximum resolved wavenumber). The Taylor-Reynolds number $R e_{\lambda}=2 E \sqrt{5 /(3 \nu \epsilon)}=196$, where $E$ is the total kinetic energy. The compensated energy spectrum from such a simulation shows an inertial range which is certainly less than a decade but still resolved (see, e.g., Fig. 1 (a) for $N=512$ and $D=3.00$ in Ref. [5]).

To identify vortical and straining structures in the flow, we use the second invariant of the velocity gradient tensor $\mathcal{Q}=\left(R^{2}-S^{2}\right) / 2$ [27-29], defined through the velocity gradient tensor $\mathcal{A}=\tau_{\eta} \nabla \boldsymbol{u}$ (normalized by the Kolmogorov time $\tau_{\eta}=(\nu / \epsilon)^{1 / 2}$ ) which yields the symmetric strain rate tensor $\mathcal{S}=\left(\mathcal{A}+\mathcal{A}^{\mathrm{T}}\right) / 2$ and the anti-symmetric rotation rate tensor $\mathcal{R}=\left(\mathcal{A}-\mathcal{A}^{\mathrm{T}}\right) / 2$. Regions where $\mathcal{Q}>0(\mathcal{Q}<0)$ are dominated by vorticity (irrotational strain) [25. Figure 1 presents contours of large positive and negative values of $\mathcal{Q}$, which reveal characteristic vortex-strain wormrolls.

We introduce in the flow $10^{6}$ identical particles, each having a sub-Kolmogorov radius $a=\eta / 3$ and a density $\rho_{p}$ much larger than that of the carrier-fluid $\rho_{f}$. The particles occupy a small volume fraction of $\mathcal{O}\left(10^{-4}\right)$ and their influence on the flow is negligible. Since the Reynolds number associated with their slip velocities is small, and $\rho_{p} \gg \rho_{f}$, the evolution of particle trajectories $\boldsymbol{X}_{p}(t)$ is determined by the simplified Maxey-Riley equations [30 33]:

$$
\frac{d \boldsymbol{X}_{p}}{d t}=\boldsymbol{V}_{p}, \quad \frac{d \boldsymbol{V}_{p}}{d t}=-\frac{1}{\tau_{p}}\left[\boldsymbol{V}_{p}-\boldsymbol{u}\left(\boldsymbol{X}_{p}, t\right)\right]
$$


where $\tau_{p}=2 a^{2} \rho_{p} /\left(9 \nu \rho_{f}\right)$, the particle relaxation time, yields the Stokes number $\left(S t=\tau_{p} / \tau_{\eta}\right)$, which provides a non-dimensional measure of the particle's inertia. We consider several families of particles, with St ranging from 0 to 16.75 and use an exponential integration scheme [34. The case of tracers $S t=0$ is handled separately by using a second order Runge-Kutta time-stepper. The fluid velocity at the particle position is obtained via fourth-order B-spline interpolation [35].

It is important to note that the particle radius influences particle transport in two distinct ways: It sets the value of $S t$, while also determining the separation between particle centers at collision. As we are considering heavy particles $\left(\rho_{p} / \rho_{f}>1\right)$, the radius must be very small for small values of $S t$. This makes the gathering of collision statistics in this dilute suspension increasingly impractical as $S t \rightarrow 0$, unless we consider an artificially enlarged collision radius. Indeed, for tracers, by definition, any choice of radius is arbitrary. Our choice of $\eta / 3$ is physically consistent for particles with $S t>0.1$. However, for smaller $S t$, this particle radius would imply $\rho_{p} / \rho_{f}<1$ which contradicts our heavy particle assumption. Indeed, to accurately describe particle motion, the simplified Maxey-Riley equations (2) require $\rho_{p} / \rho_{f}>O\left(10^{2}\right)$ [31 33. Nevertheless, it is convenient to consider this enlarged collision radius, solely for the purpose of detecting collisions. As a check, we actually performed simulations with particle radius $\eta / 10$ (where the problem is mitigated) and found our results unchanged. This can be rationalised by noting that particles move ballistically in the small distance which separates the $\eta / 3$ and the more realistic $\eta / 10$ radii and hence make our collision results insensitive to the precise choice of the radius. Nevertheless, we do report results for the larger radius because the collision statistics are much poorer with the smaller radius.

After the randomly-seeded particles have settled into a statistically stationary distribution, we begin detecting collisions using an algorithm similar to that in Ref. [21]. After a collision is detected, the two particles are allowed to move past each other without any modification to their trajectories. This ghost collision approach is a standard approximation that, by ignoring coalescence, allows one to measure collision rates while the particle distribution remains in a statistically stationary state. This feature allows us to identify and compare the regions of the flow where most of the collisions occur to those regions where most of the particles reside. Of course, because particles never coalesce, the collision rates are over-predicted (this issue is less severe for dilute systems, such as the one we consider here). We do not expect this to impact our conclusions, however, as our study is based on identifying where collisions occur, which depends on the relative values of the collision rate in different regions of the flow and not on the absolute values.

The rate of collisions depends, of course, on the relative velocity of particles at contact [3639. For tracers $(S t=0)$ or nearly-tracer particles $(S t \gtrsim 0)$, this is determined by fluid velocity gradients $\left(\propto \tau_{\eta}^{-1}\right)$, which increase in magnitude as the flow becomes more turbulent. This picture, underlying the work of Saffman and Turner [40], is blind to flow structures: It disregards whether the local velocity gradient arises from rotation or strain.

Inertial particles $(S t>0)$, e.g., droplets in air, preferentially concentrate, thereby increasing their local number density [21]. They can also attain relative velocities much larger than that of the underlying flow. Dubbed the sling effect [41], these events correspond to the formation of singularities or caustics in the particle velocity field [42, 43]. Although clustering and caustics have been tied to the centrifugal ejection of heavy particles out of vortices, they also occur in smooth random flows that are devoid of structure [44 48]. Consequently, the presence of these effects does not necessarily imply that collisions sense the structures of turbulence.

To unambiguously determine the influence of the local flow field, we must begin with tracers which remain uniformly distributed in space. To allow for collisions, the radii of these particles are kept (artificially) finite, while their inertia is ignored. According to the Saffman-Turner theory [40], collisions should occur uniformly between any two regions that possess the same velocity gradient magnitude, regardless of whether these regions are vortical or straining. We now examine this 

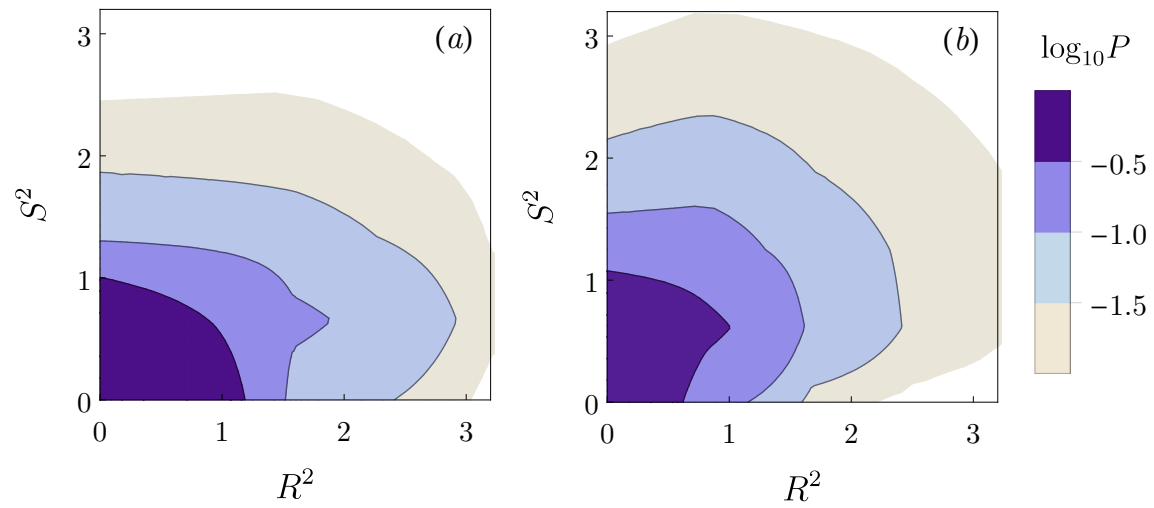

FIG. 2. Bi-probability distribution functions $P\left(R^{2}, S^{2}\right)$ for inertia-less (tracer) particles, corresponding to the values of $R^{2}$ and $S^{2}$ sampled by (a) particles and (b) collision locations, which show the disproportionate bias towards collisions in strain-dominated regions.
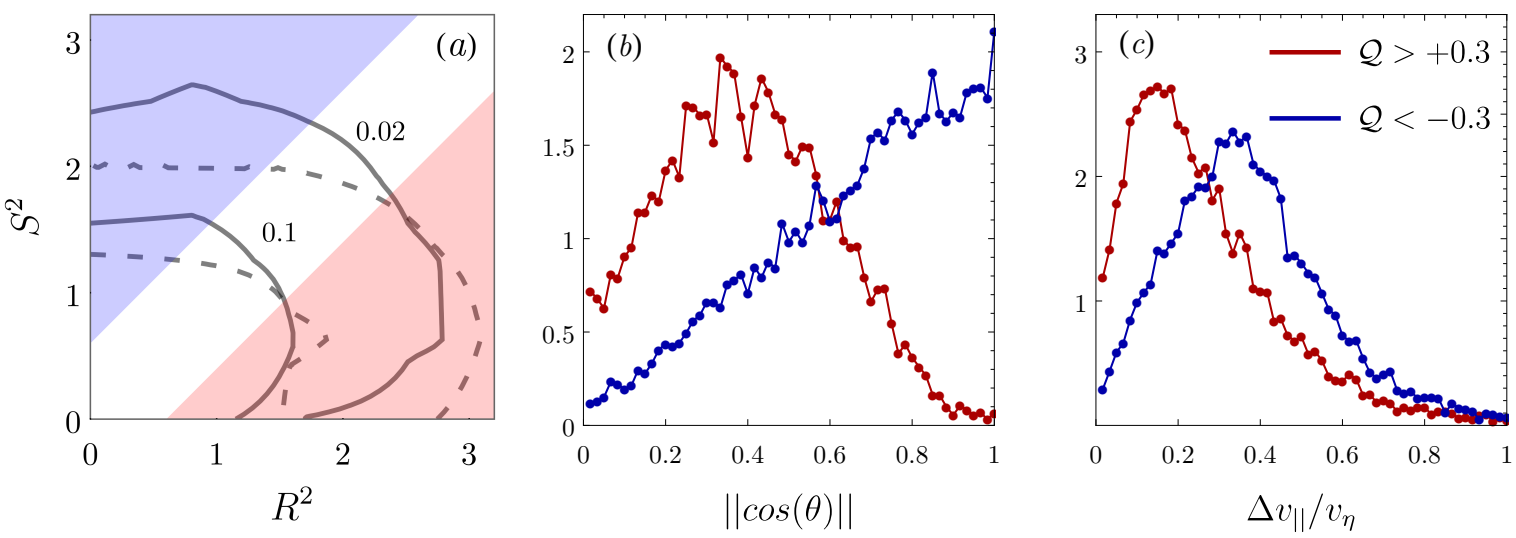

FIG. 3. (a) Contours corresponding to $P\left(R^{2}, S^{2}\right)=0.02,0.1$, sampled by inertia-less particles (dashed) and their collisions (solid). The blue and red shaded portions correspond to $\mathcal{Q}<-0.3$ and $\mathcal{Q}>+0.3$, and indicate regions dominated by strain and vorticity, respectively. Conditional probability distributions of (b) the cosine of the angle of collision $\theta$, for data corresponding to the shaded portions of panel (a), and (c) the approach velocity at collision $\Delta v_{\|}$, normalized by the Kolmogorov velocity $v_{\eta}$. These panels clearly illustrate why straining regions are more effective at generating collisions.

hypothesis, bearing in mind that a discrepancy will implicate flow structures that are intrinsically more effective at causing collisions.

Towards this end, we calculate the values of $R^{2}$ and $S^{2}$ along particle trajectories, as well as at collision locations. The results for inertia-less particles are presented as joint probability distributions functions $P\left(R^{2}, S^{2}\right)$ in Fig. 2 and Fig. $2 \mathrm{~b}$, respectively. It is immediately clear that collisions under-sample vortical regions $\left(R^{2}>S^{2}\right)$ and over-sample straining regions $\left(S^{2}>R^{2}\right)$, relative to where particles reside. This is also seen in Fig. 3a, which overlays contours of $P\left(R^{2}, S^{2}\right)=$ 0.1 and 0.02 , for particles (dashed) and collisions (solid). The strain (vorticity) dominated portion of this plot is shaded in blue (red), and corresponds to $\mathcal{Q}<-0.3(\mathcal{Q}>+0.3)$.

This surprising result is an outcome of the distinct flow topologies of these regions which cause particles to approach each other differently. Fig. $3 \mathrm{~b}$ presents the distribution of the cosine of the collision angle $(\theta)$, for straining (blue) and vortical (red) regions. $\theta$ is defined as the angle between the relative velocity vector $\left(V_{p 1}-V_{p 2}\right)$ and the separation vector $\left(X_{p 1}-X_{p 2}\right)$ at collision. Particles 

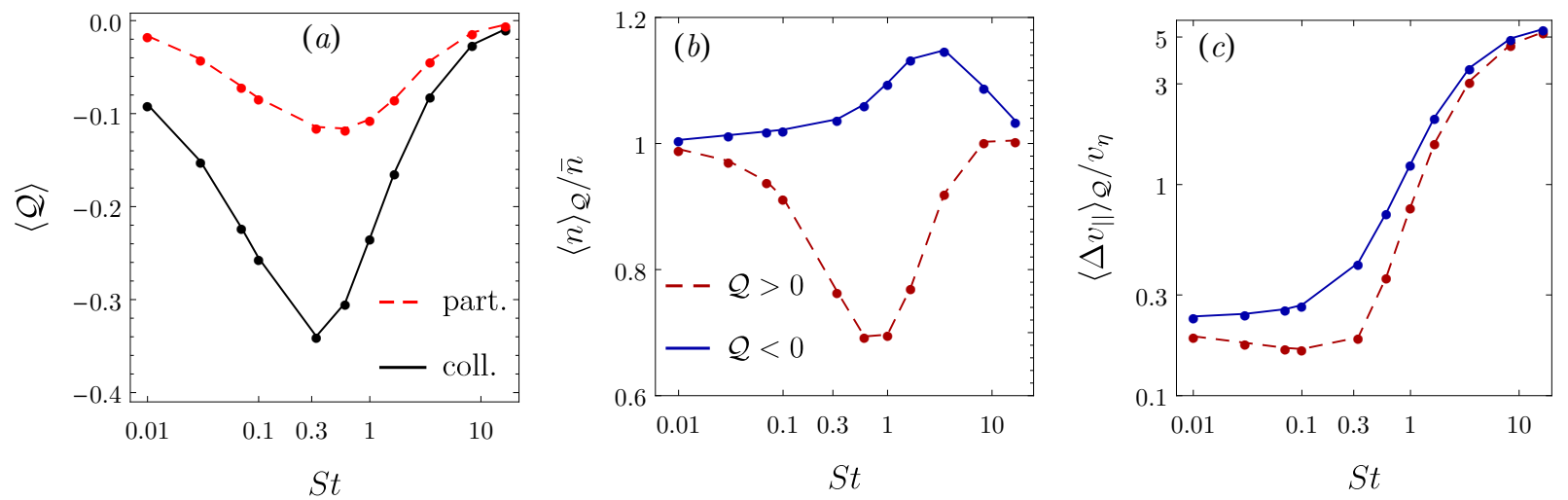

FIG. 4. (a) Average $\mathcal{Q}$, sampled by particles (red-dashed) and collisions (black-solid), as a function of St. (b) Average particle number density in vortical and straining regions (positive and negative $\mathcal{Q}$ ), plotted as a function of $S t$, and normalized by the domain-average number density $\bar{n}$. (c) Average approach velocity for collisions in vortical and straining regions, normalized by $v_{\eta}$

in straining regions tend to collide in a head-on or rear-end manner $(\theta \approx 0$ or $\pi)$. In either case, a large fraction of the velocity difference between particles contributes to their rate of approach or collision velocity $\left(\Delta v_{\|}\right)$. On the other hand, particles in vortices undergo collisions that are closer to being side-on, in which case the separation vector is nearly perpendicular to the relative velocity. This results in lower approach velocities in vortical regions, as shown in Fig. 3r. Consequently, over a given time interval, fewer particles will collide in vortical regions compared to straining regions with the same magnitude of the velocity gradient and particle number density.

How does particle inertia affect this picture? Figure 4 presents the average value of $\mathcal{Q}$ sampled by particles (dashed-red) and their collision locations (solid-black) as a function of $S t$. At $S t=0$, $\langle\mathcal{Q}\rangle$ is 0 for particles and -0.04 for collisions. Remarkably, this offset is strongly amplified by inertia and reaches a maximum around $S t \approx 0.3$, beyond which particles begin to de-correlate from the underlying flow and eventually collide uniformly. The preference of inertial particles $(S t>0)$ to collide in straining regions has been reported previously by Perrin and Jonker [49, 50]. Our results demonstrate that this effect is not fundamentally tied to particle inertia, but rather is an amplification of a difference that exists even for inertia-less tracers, raising the question: How does particle inertia selectively enhance collisions in straining regions?

One possible explanation is provided by preferential concentration: Heavy particles are centrifuged out of rotational regions, and thus tend to accumulate in straining zones just outside vortices [43]. This causes the number density to increase in straining regions, at the expense of vortical zones, as shown in Fig. $4 \mathrm{~b}$. Here, $n$ is a coarse-grained number density, obtained by dividing the domain into bins of size $20 \eta$. The average $\mathcal{Q}$ in each bin is used to distinguish between vortical $(\mathcal{Q}>0)$ and straining regions $(\mathcal{Q}<0)$ and obtain the conditionally averaged number density. All else being equal, higher number densities imply larger collision rates [20]. However, we see that the maximum difference in number densities occurs near $S t \approx 1$, which is not where the maximum difference in $\langle\mathcal{Q}\rangle$ is seen (Fig. 4a). Hence another mechanism must be involved.

Inertia is also known to increase the relative velocity between neighboring particles [36 38], which should result in higher collision velocities. On examining this effect in straining and vortical regions separately, we find that it is stronger in straining regions and, in fact, has no impact on vortical regions for small St. This is demonstrated in Fig. 4k, which presents the average values of $\Delta v_{\|}$, conditioned on $\mathcal{Q}$. It appears that head-on (or rear-end) collisions, which prevail in straining zones, are more amenable to being sped-up by inertia than side-on collisions. Notably, the 

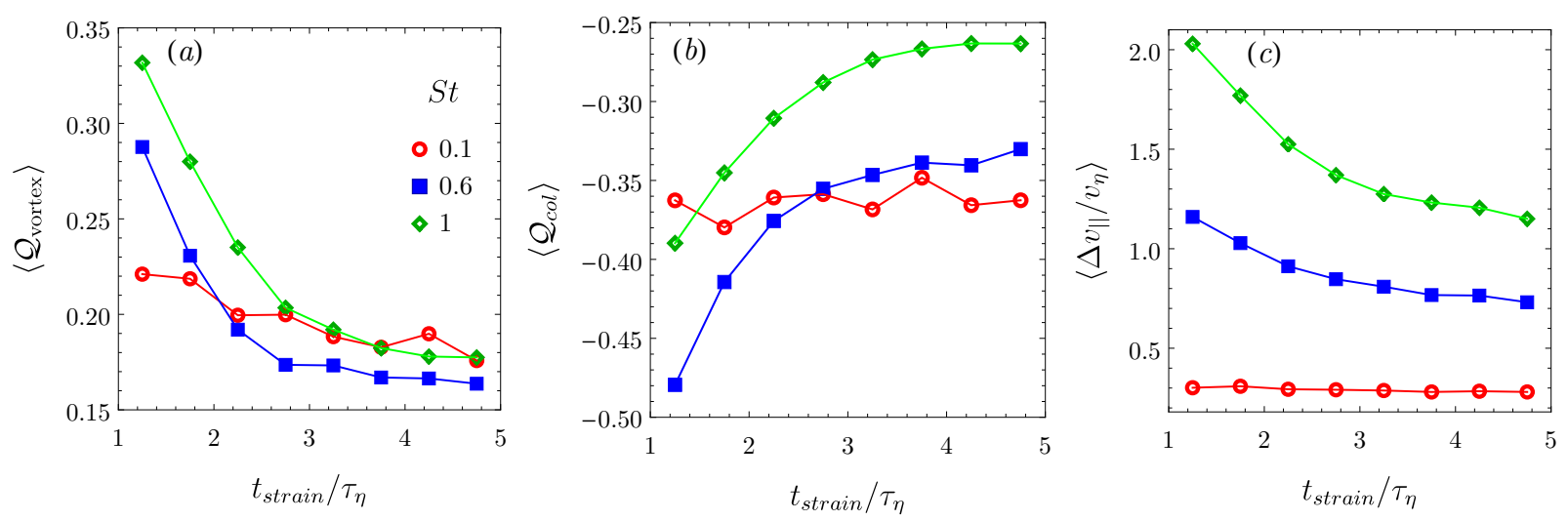

FIG. 5. Representative plots of (a) the maximum value of $\mathcal{Q}$ sampled by the particle inside the vortical region; (b) the average value of $\mathcal{Q}$ at collision; and (c) the corresponding approach velocity at collisions obtained from Lagrangian tracking of particles that collide in straining regions $(\mathcal{Q}<0)$ after leaving a vortical region and conditionally averaged on the time taken to collide after entering the straining region $\left(t_{\text {strain }}\right)$. These plots clearly illustrate the singular importance of vortex-strain worm-rolls as discussed in the text.

maximum difference between collision velocities occurs near $S t \approx 0.3$, which matches well with the maximum difference in $\langle\mathcal{Q}\rangle$ (Fig. 4 a). Thus, larger approach rates, rather than number densities, appear to be the primary reason for the effectiveness of straining regions in creating collisions.

Thus far, we have considered vortical and straining regions individually. Particle inertia, however, permits structures within a distance of $\tau_{p} V_{p}$ to influence a collision. This raises the possibility of vortical and straining regions conspiring to enhance collisions, especially for moderately large St. For example, the geometry of vortex-strain worm-rolls (Fig. 1) will cause particles in intense vortex tubes to be rapidly ejected into strong straining sheets, where they are very likely to collide.

We search for evidence of this effect by tracing, backward in time, all particles that collide in straining regions. For the subset that do have at least one particle originating from a vortical region $(Q>0)$, we record (i) the time taken to collide after leaving the vortical region and entering the straining zone $\left(t_{\text {strain }}\right)$, as indicated by $\mathcal{Q}$ changing sign; (ii) the strength of the vortex, measured in terms of the maximum positive value of $\mathcal{Q}$ sampled inside the vortical region ( $\left.\mathcal{Q}_{\text {vortex }}\right)$; (iii) the intensity of straining at the collision location $\left(\mathcal{Q}_{c o l}\right)$; and (iv) the collision velocity. While measuring the vortex strength, we only back-track for a time of $3 \tau_{\eta}$ within the vortical region, to ensure that the $\mathcal{Q}$ values obtained are relevant to the subsequent collision. The conclusions are insensitive to the exact value of this threshold. To facilitate back-tracking, we store the values of $\mathcal{Q}$ along all particle trajectories, at time intervals of $\tau_{\eta} / 6$, for the entire duration of the simulations. This avoids having to store the velocity field at each time step, for integrating the particle motion backward in time (as done in [51]), which, given the rarity of collisions and the consequent long simulation time, would require prohibitively large amounts of computer storage.

Figure 5 presents the results of this backward-in-time Lagrangian calculation, conditionally averaged on the time taken to collide after leaving a vortical region, $t_{\text {strain }}$, for $S t=1,0.6$ and 0.1. The data for moderately large $S t$ (1 and 0.6), reveal the impact of vortex-strain worm-rolls. Particles that collide quickly (small $t_{\text {strain }}$ ), are found to originate from more intense vortical regions (Fig. 5a) and to collide in stronger straining regions (Fig. 5b). This signature weakens considerably for less inertial $(S t=0.1)$ particles which are mildly ejected and relax faster to the local straining flow.

The collision velocities for small $t_{\text {strain }}$ are also systematically larger (Fig. 55). The standard deviation about each data point (not shown for clarity) is of the order of the average value. Thus, 
several small $t_{\text {strain }}$ collisions have very large collision velocities, indicative of caustics/sling events, which are known to dominate the collision rate for $S t>0.5$ [20, 24]. Traditionally, these have been linked to rapid ejection from vortices [41]. Our results indicate that this is only half the story: Straining sheets which envelope strong vortices also contribute to generating violent collisions and enhancing collision rates.

All this leads one to ask how collisions are affected when structures change. The influence of $R e_{\lambda}$ is particularly important to consider, as the estimated values for natural flows are orders of magnitude larger than what can be attained in simulations [17, 18. Increasing $R e_{\lambda}$ results in higher intermittency, which translates into more intense structures (see, e.g., Ref. [52] for a similar study of a model stretched-vortex), but which occupy smaller volumes. These competing effects are known to produce a non-monotonic variation of particle clustering [53. For collisions in particular, we have checked explicitly through simulation that as we increase the Reynolds numbers from $R e_{\lambda}=69$ to 196, unsurprisingly, the differences between vortical and straining regions magnifies; therefore our conclusions, substantiated in this paper from simulations with $R e_{\lambda}=196$ hold. Nevertheless, we should keep in mind that $R e_{\lambda}=196$ is still a reasonably modest Reynolds number and far from those seen in atmospheric conditions. Therefore, it remains to be seen in a systematic way how this phenomenon is affected in a higher Reynolds number flow. However, given our present understanding of the effect of increasing Reynolds numbers on turbulent structures, we expect that the central results of our work will remain unchanged and the effect that we elucidate will only show up more clearly.

Flow structures can also be significantly modified by new physical interactions, for example, condensation of water vapour on cloud droplets, which releases latent heat and energizes small scales [54, and elastic feedback from polymers that suppresses small-scale motions [55, 56]. Studying collisions in these complex flows is left for future work. Furthermore, in order to clearly examine the effect reported in this work, we have neglected the role of gravity. We know that in the limit of small Froude numbers (ratio of turbulent to gravitational acceleration) [57, heavy droplets settle in a way where the fluid structures are sampled differently from the case when Froude numbers are large. In such low Froude number flows, it remains to be seen how the mechanism described in this paper is modified.

Before we conclude, it is essential to place our work in the context of turbulent transport problems - a canonical example being that of rain-initiation in warm clouds - which have application across the areas of non-equilibrium statistical physics, geophysics, oceanography, astrophysics and atmospheric sciences. Understanding these problems demands not only an appreciation of how fast droplets sediment, collide and coalesce (in which tremendous advances have been made in recent years) but also knowledge of where such processes are most likely to occur. This paper, therefore, contributes to a fuller understanding of this question.

\section{ACKNOWLEDGMENTS}

We thank Jérémie Bec, S. Ravichandran and Siddhartha Mukherjee for useful suggestions and discussions, which were facilitated in part by a program organized at ICTS: Turbulence from Angstroms to Light Years (ICTS/Prog-taly2018/01). The simulations were performed on the ICTS clusters Mowgli and Mario as well as the work stations from the project ECR/2015/000361: Goopy 
and Bagha. SSR acknowledges DST (India) project ECR/2015/000361 for financial support.

[1] U. Frisch, Turbulence: The legacy of A N Kolmogorov (Cambridge University Press, Cambridge, UK, 1996).

[2] A. Tsinober, An informal conceptual introduction to turbulence (Springer, New York, USA, 2009).

[3] J. Schumacher, R. M. Kerr, and K. Horiuti, "Structure and dynamics of vorticity in turbulence," in Ten Chapters in Turbulence, edited by P. A. Davidson, Y. Kaneda, and K. R. Sreenivasan (Cambridge University Press, 2012) p. 4386.

[4] U. Frisch, A. Pomyalov, I. Procaccia, and S. S. Ray, "Turbulence in noninteger dimensions by fractal fourier decimation," Phys. Rev. Lett. 108, 074501 (2012).

[5] M. Buzzicotti, A. Bhatnagar, L. Biferale, A. S. Lanotte, and S. S. Ray, "Lagrangian statistics for navier-stokes turbulence under fourier-mode reduction: fractal and homogeneous decimations," New J. Phys. 18, 113047 (2016).

[6] A. S. Lanotte, S. K. Malapaka, and L. Biferale, "On the vortex dynamics in fractal fourier turbulence," Eur. Phys. J. E 39, 49 (2016).

[7] B. W. Zeff, D. D. Lanterman, R. McAllister, R. Roy, E. J. Kostelich, and D. P. Lathrop, "Measuring intense rotation and dissipation in turbulent flows," Nature 34, B479-B498 (2003).

[8] R. Pandit, P. Perlekar, and S. S. Ray, "Statistical properties of turbulence: An overview," PramanaJournal of Physics 73, 157 (2009).

[9] J. Schumacher, B. Eckhardt, and C. R. Doering, "Extreme vorticity growth in navier-stokes turbulence," Phys. Lett. A 374, 861-865 (2010).

[10] P. Gotzfried, B. Kumar, R. A. Shaw, and J. Schumacher, "Droplet dynamics and fine-scale structure in a shearless turbulent mixing layer with phase changes," J. Fluid Mech. 814, 452483 (2017).

[11] M. Guala, A. Liberzon, A. Tsinober, and W. Kinzelbach, "An experimental investigation on lagrangian correlations of small-scale turbulence at low reynolds number," J. Fluid Mech. 574, 405427 (2007).

[12] P. E. Hamlington, J. Schumacher, and W. J. A. Dahm, "Local and nonlocal strain rate fields and vorticity alignment in turbulent flows," Phys. Rev. E 77, 026303 (2008).

[13] S. S. Ray, "Thermalised solutions, statistical mechanics and turbulence: An overview of some recent results," Pramana-Journal of Physics 84, 395 (2015).

[14] M. Wilczek, "New insights into the fine-scale structure of turbulence," J. Fluid Mech. 784, 14 (2015).

[15] J. M. Lawson and J. R. Dawson, "On velocity gradient dynamics and turbulent structure," J. Fluid Mech. 780, 6098 (2015).

[16] S. S. Ray, "Non-intermittent turbulence: Lagrangian chaos and irreversibility," Phys. Rev. Fluids (Rapid) 18, 072601(R) (2018).

[17] J. J. Lissauer, "Planet formation," Annu. Rev. Astron. and Astrophys. 31, 129-172 (1993).

[18] W. W. Grabowski and L.-P. Wang, "Growth of cloud droplets in a turbulent environment," Annu. Rev. Fluid Mech. 45, 293-324 (2013).

[19] S. Chen, M.-K. Yau, P. Bartello, and L. Xue, "Bridging the condensation-collision size gap: a direct numerical simulation of continuous droplet growth in turbulent clouds," Atmos. Chem. Phys. 18, 72517262 (2018).

[20] A. Pumir and M. Wilkinson, "Collisional aggregation due to turbulence," Annu. Rev. Conden. Ma. P. 7, 141-170 (2016).

[21] S. Sundaram and L. R. Collins, "Collision statistics in an isotropic particle-laden turbulent suspension. part 1. direct numerical simulations," J. Fluid Mech. 335, 75109 (1997).

[22] J. Bec, S. S. Ray, E. W. Saw, and H. Homann, "Abrupt growth of large aggregates by correlated coalescences in turbulent flow," Phys. Rev. E 93, 031102(R) (2016).

[23] R. Onishi, K. Matsuda, and K. Takahashi, "Lagrangian tracking simulation of droplet growth in turbulenceturbulence enhancement of autoconversion rate," J. Atmos. Sci. 72, 2591-2607 (2015).

[24] M. Voßkuhle, A. Pumir, E. Lévêque, and M. Wilkinson, "Prevalence of the sling effect for enhancing collision rates in turbulent suspensions," J. Fluid Mech. 749, 841852 (2014).

[25] P. J. Ireland, A. D. Bragg, and L. R. Collins, "The effect of reynolds number on inertial particle dynamics in isotropic turbulence. part 1. simulations without gravitational effects," J. Fluid Mech. 
796, $617658(2016)$.

[26] C. Canuto, M. Y. Hussaini, A. Quarteroni, and T. A. Zang, Spectral methods: fundamental in single domains (Springer-Verlag, Berlin, Germany, 2006).

[27] Y. Dubief and F. Delcayre, "On coherent-vortex identification in turbulence," J. Turbul. 1, N11 (2000).

[28] H. M. Blackburn, N. N. Mansour, and B. J. Cantwell, "Topology of fine-scale motions in turbulent channel flow," J. Fluid Mech. 310, 269292 (1996).

[29] M. S. Chong, A. E. Perry, and B. J. Cantwell, "A general classification of threedimensional flow fields," Phys. Fluids A 2, 765-777 (1990).

[30] S. Ravichandran, P. Deepu, and R. Govindarajan, "Clustering of heavy particles in vortical flows: a selective review," Sādhanā 42, 597-605 (2017).

[31] V. Armenio and V. Fiorotto, "The importance of the forces acting on particles in turbulent flows," Phys. Fluids 13, 2437-2440 (2001).

[32] M. A. T. van Hinsberg, H. J. H. Clercx, and F. Toschi, "Enhanced settling of nonheavy inertial particles in homogeneous isotropic turbulence: The role of the pressure gradient and the basset history force," Phys. Rev. E 95, 023106 (2017).

[33] M. van Aartrijk and H. J. H. Clercx, "Vertical dispersion of light inertial particles in stably stratified turbulence: The influence of the basset force," Phys. Fluids 22, 013301 (2010).

[34] P. J. Ireland, T. Vaithianathan, P. S. Sukheswalla, B. Ray, and L. R. Collins, "Highly parallel particleladen flow solver for turbulence research," Comput. Fluids 76, 170 - 177 (2013).

[35] M. A. T. van Hinsberg, J. H. M. T. Boonkkamp, F. Toschi, and H. J. H. Clercx, "On the efficiency and accuracy of interpolation methods for spectral codes," SIAM J. Sci. Comput. 34, B479-B498 (2012).

[36] E.-W. Saw, G. P. Bewley, E. Bodenschatz, S. S. Ray, and J. Bec, "Extreme fluctuations of the relative velocities between droplets in turbulent airflow," Phys. Fluids 26, 111702 (2014).

[37] M. James and S. S. Ray, "Enhanced droplet collision rates and impact velocities in turbulent flows: The effect of poly-dispersity and transient phases," Sci. Rep. 7, 12231 (2017).

[38] A. Bhatnagar, K. Gustavsson, and D. Mitra, "Statistics of the relative velocity of particles in turbulent flows: Monodisperse particles," Phys. Rev. E 97, 023105 (2018).

[39] A. Bhatnagar, K. Gustavsson, B. Mehlig, and D. Mitra, "Relative velocities in bidisperse turbulent aerosols: Simulations and theory," Phys. Rev. E 98, 063107 (2018).

[40] P. G. Saffman and J. S. Turner, "On the collision of drops in turbulent clouds," J. Fluid Mech. 1, 1630 (1956).

[41] G. Falkovich, A. Fouxon, and M. G. Stepanov, "Acceleration of rain initiation by cloud turbulence," Nature 419, 151-154 (2002).

[42] M. Wilkinson, B. Mehlig, and V. Bezuglyy, "Caustic activation of rain showers," Phys. Rev. Lett. 97, 048501 (2006).

[43] S. Ravichandran and R. Govindarajan, "Caustics and clustering in the vicinity of a vortex," Phys. Fluids 27, 033305 (2015).

[44] J. Bec, A. Celani, M. Cencini, and S. Musacchio, "Clustering and collisions of heavy particles in random smooth flows," Phys. Fluids 17, 073301 (2005).

[45] M. Wilkinson, B. Mehlig, S. Östlund, and K. P. Duncan, "Unmixing in random flows," Phys Fluids 19, 113303 (2007).

[46] J. Bec, S. Musacchio, and S. S. Ray, "Sticky elastic collisions," Phys. Rev. E 87, 063013 (2013).

[47] K. Gustavsson and B. Mehlig, "Distribution of velocity gradients and rate of caustic formation in turbulent aerosols at finite kubo numbers," Phys. Rev. E 87, 023016 (2013).

[48] K. Gustavsson and B. Mehlig, "Relative velocities of inertial particles in turbulent aerosols," J. Turbul. 15, 34-69 (2014).

[49] V. E. Perrin and H. J. J. Jonker, "Preferred location of droplet collisions in turbulent flows," Phys. Rev. E 89, 033005 (2014).

[50] V. E. Perrin and H. J. J. Jonker, "Effect of the eigenvalues of the velocity gradient tensor on particle collisions," J. Fluid Mech. 792, 3649 (2016).

[51] M. Wan, Z. Xiao, C. Meneveau, G. L. Eyink, and S. Chen, "Dissipation-energy flux correlations as evidence for the lagrangian energy cascade in turbulence," Phys. Fluids 22, 061702 (2010).

[52] L. Agasthya, J. R. Picardo, S. Ravichandran, R. Govindarajan, and S. S. Ray, "Droplet collisions in turbulence: Insights from a burgers vortex," arXiv e-prints (2018), arXiv:1811.04486

[53] R. Onishi and J. C. Vassilicos, "Collision statistics of inertial particles in two-dimensional homogeneous 
isotropic turbulence with an inverse cascade," J. Fluid Mech. 745, 279299 (2014).

[54] S. Ravichandran and R. Govindarajan, "Vortex-dipole collapse induced by droplet inertia and phase change," J. Fluid Mech. 832, 745776 (2017).

[55] A. Liberzon, M. Guala, W. Kinzelbach, and A. Tsinober, "On turbulent kinetic energy production and dissipation in dilute polymer solutions," Phys. Fluids 18, 125101 (2006).

[56] P. Perlekar, D. Mitra, and R. Pandit, "Direct numerical simulations of statistically steady, homogeneous, isotropic fluid turbulence with polymer additives," Phys. Rev. E 82, 066313 (2010).

[57] J. Bec, H. Homann, and S. S. Ray, "Gravity-driven enhancement of heavy particle clustering in turbulent flow," Phys. Rev. Lett. 112, 184501 (2014). 